19 Revue d'histoire du XIXe siècle

Société d'histoire de la révolution de 1848 et des

révolutions du XIXe siècle

$29 \mid 2004$

Varia

Pierre Milza, Napoléon III, Paris, Éditions Perrin, 2004, 706 p.

Fabien Cardoni

(2) OpenEdition

Journals

Édition électronique

URL : http://journals.openedition.org/rh19/711

DOI : 10.4000/rh19.711

ISSN : $1777-5329$

Éditeur

La Société de 1848

Édition imprimée

Date de publication : 1 décembre 2004

Pagination : 182-185

ISSN : 1265-1354

Référence électronique

Fabien Cardoni, « Pierre Milza, Napoléon III, Paris, Éditions Perrin, 2004, 706 p. », Revue d'histoire du XIXe siècle [En ligne], 29 | 2004, mis en ligne le 29 juin 2005, consulté le 22 septembre 2020. URL http://journals.openedition.org/rh19/711 ; DOI : https://doi.org/10.4000/rh19.711

Ce document a été généré automatiquement le 22 septembre 2020.

Tous droits réservés 


\title{
Pierre Milza, Napoléon III, Paris, Éditions Perrin, 2004, 706 p.
}

\author{
Fabien Cardoni
}

1 La dernière biographie de Napoléon III date de 1990 (Philippe Séguin, Louis Napoléon le Grand, Paris, Grasset). La biographie de Louis Girard - dont Pierre Milza affirme qu'elle est la meilleure (chapitre 1, note 10) - a été rééditée trois fois depuis 1986 (dernière édition chez Hachette Littératures en 2002). Une nouvelle étude est donc la bienvenue, participant par là au renouveau historiographique du Second Empire entamé il y a une dizaine d'années déjà.

Dans son livre, Pierre Milza fait bien ressortir tout d'abord que cet empire n'est pas simplement une parenthèse entre deux républiques, mais bien une «étape » dans la démocratisation de la France et notamment une période qui « a familiarisé les Français avec le vote" (p.646). Pierre Milza souligne également que le Second Empire «appartient à la galaxie démocratique » (p.630) et que "rares sont les dictatures européennes dont l'évolution s'est opérée comme celle-ci dans le sens de la libéralisation» (p. 645). L'auteur expose par ailleurs avec finesse que «la dénonciation du césarisme, réel ou supposé, appartient à la culture de la République parlementaire » (p.620). Pierre Milza, qui se définit comme « un républicain qui ne nourrit aucune nostalgie à l'égard de l'empire» (p.648), nous offre en outre un portrait sans complaisance de la forte personnalité de Louis-Napoléon. L'auteur nous rappelle que ce dernier n'a guère connu le fondateur de la dynastie - à la fois son oncle et son grandpère - que pendant les Cent-Jours. Le premier Napoléon restera en revanche toujours son mentor. L'auteur évoque tour à tour les années d'exil de Louis-Napoléon en Suisse et en Angleterre, son engagement pour la Romagne, ainsi que ses tentatives de coup d'État manquées de Strasbourg et Boulogne qui, d'après Pierre Milza, «n'ont pas été aussi dérisoires qu'on l'a dit » (p. 78). Cette étude démontre avec habileté l'importance des six années d'emprisonnement (L'« université de Ham», p. 115 et suiv.) dans la formation morale et intellectuelle du futur empereur. L'auteur évoque bien entendu ensuite l'évasion rocambolesque de la prison de Ham. Pierre Milza nous informe en outre constamment sur les - innombrables - relations amoureuses de Louis-Napoléon, 
sans verser cependant dans l'anecdotique. Le chapitre 10 nous présente un bon panorama du personnel politique contemporain et des proches de l'empereur, où l'auteur insiste par ailleurs sur la dimension sociale de la politique de l'empereur et sur sa capacité à imposer des réformes que son entourage désapprouve parfois. Pierre Milza souligne également le rôle personnel du souverain dans la politique extérieure. Les chapitres abordant les relations internationales sont parmi les meilleurs pour leur clarté. Du reste, Pierre Milza parvient avec talent à étudier en parallèle les politiques intérieure et extérieure. On sent que l'auteur a pris du plaisir à raconter ce destin hors du commun. Le style est très agréable, même si quelques formules sont plus ou moins bien senties : « Il renvoie Guizot à ses chères études historiques » (p. 138), les « râteliers nationaux» (p. 144), "populiste bon teint » à propos de Louis-Napoléon (p. 200), «dictature municipale » d'Haussmann à Paris, «alliance du Trône et de la Bourse » (p. 645). À propos de la crise orientale de 1840, Pierre Milza annonce que «Thiers se déclara prêt à l'épreuve de force. Il fit fortifier Paris et procéda à une mobilisation partielle» (p. 101), comme si les deux actions étaient synchrones. Il est par ailleurs exagéré d'affirmer que l'armée du Second Empire «était faite pour le temps de paix, pour les scintillantes revues du Carrousel ou du Champ-de-Mars, ou pour briser les tentatives insurrectionnelles » (p. 311). Enfin, on peut lire (p. 547) : « Blanqui a rédigé à leur intention une sorte de manuel de l'insurgé : Instruction pour une prise d'armes, dont il aura l'occasion de mesurer l'inefficacité lors des événements de septembre $1870 »$. Or la journée du 4 septembre n'est pas une lutte armée. Page 119, une erreur de style - ou d'attention - laisse penser que l'abolition de l'esclavage date de 1840. Quelques pages plus loin (p. 256), Pierre Milza évoque les effectifs des forces de l'ordre pour relativiser - avec justesse - un Empire souvent perçu comme un État policier. Qu'il nous soit permis de préciser ici que s'il y a bien, comme on peut le lire, une régression des effectifs totaux de la gendarmerie entre le début et la fin du règne, elle est la conséquence de la disparition des formations gendarmiques de la garde impériale. Le nombre de gendarmes départementaux reste lui stable entre 1852 et 1870 et la garde de Paris, qui fait partie intégrante de cette arme, voit ses effectifs augmenter. En outre, la police parisienne et celle de la province connaissent une forte croissance durant le Second Empire.

3 À plusieurs reprises, Pierre Milza laisse le lecteur sur sa faim. En effet, il ne présente rien de nouveau sur la question de la paternité de Louis-Napoléon (pp. 14-16) ou sur l'éventuelle appartenance de ce jeune Bonaparte à la Charbonnerie (pp.45-46). Le lecteur appréciera le développement sur Verdi (pp. 453-458) - dont Pierre Milza est le brillant biographe -, il s'étonnera en revanche que l'auteur consacre autant de pages au musicien qu'aux trois dernières semaines $d u$ règne $d u$ second empereur (pp. 588-593). Pierre Milza ne s'attarde guère non plus sur les écrits de jeunesse de Louis-Napoléon (pp. 120-124) et ne développe pas ces " principes que l'on retrouvera, un siècle plus tard, dans le fascisme» (p. 124). Car si l'auteur annonce (p. 465) que l'objet de son livre est la "dimension historique du personnage ", il amorce néanmoins à plusieurs occasions une mise en parallèle entre bonapartisme et fascisme (pp. 124, 157, 253-254, 259-260, 631) et entre les destins de Napoléon III et Mussolini (p. 618). Cette approche est appétissante mais l'analyse reste trop timide, même s'il faut bien entendu être "prudent» (p. 259) dans ce genre de comparaison anachronique. Pierre Milza a sans doute beaucoup à nous apprendre sur les concepts de bonapartisme, césarisme, démocratie plébiscitaire, populisme..., mais ils ne sont guère développés, y compris dans le chapitre censé les disséquer (chapitre 21 «Survie et mutations du 
bonapartisme »). Dans cette dernière partie, on survole la «légende noire" (pp. 617-620), puis l'on passe au «jugement des historiens» (pp.621-625) sans connaître celui de Pierre Milza. Ensuite, après ce bilan historiographique - où manquent cependant les travaux de Jean Tulard -, on aurait aimé lire les réflexions du spécialiste du fascisme sur les échos de ce second empire français dans l'histoire politique européenne - et bien entendu française - jusqu'à nos jours. Pourtant l'auteur, lorsqu'il analyse le régime politique et ses héritiers, ne fait que reprendre les travaux de Maurice Agulhon, René Rémond, Pierre Rosanvallon et Serge Berstein.

Sur le plan formel, les notes - rejetées à la fin de l'ouvrage, ce qui n'est jamais pratique - sont parfois incomplètes. Manquent ainsi le carton et/ou le dépôt d'archives (chap. 6 , note 1 ; chap. 1 , note 16 ; chap. 12 , note 6 ; chap. 18 , note 12 ). Faut-il voir là de simples coquilles, qui du reste se multiplient inexplicablement dans les deux cents dernières pages? Sans doute car, dans la bibliographie, l'étude de Jean Maurain sur Baroche est doublement citée et l'ouvrage de Jean-Claude Yon sur Offenbach ne comporte pas de titre. Dans le texte, certaines affirmations ne sont pas appuyées par une référence à une source imprimée ou manuscrite. De la même façon, on regrette les citations entre guillemets sans appel de note (pp. 64, 107, 110, 111, 139, 191, 253, 279, 619). Par ailleurs, l'essentiel des documents manuscrits cités sont tirés d'ouvrages imprimés. L'auteur ne cite, pour les Archives nationales, qu'un carton de la série $\mathrm{F}^{7}$, un carton de la sous-série $A B$ XIX ainsi que cinq cartons d'un seul fonds privé (correspondance de Napoléon III, 400 AP 69, 53, 43, 41, 40). Quid des fonds Maupas, Victor Noir, Rouher, Persigny, Morny, Ollivier, Pélissier, Cavaignac, Randon...? Les archives diplomatiques sont judicieusement sollicitées, mais avec une parcimonie décevante. Dans ces conditions, il n'est guère surprenant de ne pas trouver de carton d'archives dans la liste des «sources». Quant aux sources imprimées, elles sont bien entendu pléthoriques pour un tel sujet. Mais la liste des références donnée ici est trop limitée (deux pages). Dans la bibliographie, si l'auteur se réfère aux travaux les plus récents, notamment - et c'est à saluer - à des thèses inédites, il omet quelques ouvrages importants comme par exemple ceux d'Alain Dalotel, Alain Faure et JeanClaude Freiermuth, Aux origines de la Commune: le mouvement des réunions publiques à Paris 1868-1870 (Paris, Maspero, 1980, 374 p.) ou encore de Jacques de Brabant, Achille Fould et son temps (1800-1867). L'homme clef du Second Empire (Paris, CAIRN, 2001, 343 p.). L'auteur semble par ailleurs ignorer la thèse de Claude Vigoureux sur Maupas (Maupas, le préfet de police du Deux décembre, sous la direction de Jean Tulard, EPHE, 1996, 2 volumes, 159 p. et 231 p. ; édité sous le titre Maupas et le coup d'État de Louis-Napoléon, Paris, Éditions S.P.M., 2003) et ses précieuses informations sur le coup d'État recueillies dans des archives privées encore inaccessibles. Cette bibliographie ne comprend en outre que trois titres sur le coup d'État et aucun sur la guerre de 1870-1871.

5 En résumé, cette biographie devient très vite une histoire générale du Second Empire qui n'est jamais une analyse du bonapartisme. Cette étude - sans aucun doute la plus volumineuse sur Napoléon III - restera comme une bonne synthèse de l'historiographie sur le sujet, accessible au profane mais moins utile à l'initié. Ce livre est d'ores et déjà un succès de librairie. Il a reçu le prix Le Point de la biographie du festival de Nîmes 2004 ainsi que le prix des Ambassadeurs. 